\title{
Cost-effectiveness of a Province-wide Quality Improvement Initiative for Reducing Potentially Inappropriate Use of Antipsychotics in Long-Term Care in British Columbia, Canada
}

\author{
Asif Raza Khowaja ${ }^{1} \cdot$ Christina Krause $^{2} \cdot$ Colleen Kennedy $^{3} \cdot$ Ben Ridout $^{4} \cdot$ Sarah Carriere $^{5} \cdot$ Craig Mitton $^{6}$
}

Accepted: 12 April 2021 / Published online: 29 April 2021

(c) The Author(s) 2021

\begin{abstract}
Background Potentially inappropriate use of antipsychotics (PIUA) raises serious concerns about safety, quality, and cost of care for residents in long-term care (LTC).

Objective This study aimed to estimate the cost-effectiveness of the Call for Less Antipsychotics in Long-Term Care (Clear) initiative compared with the status quo (pre-Clear, baseline).

Methods A model-based cost-utility analysis, from a public-payer perspective in British Columbia, was conducted using secondary data of residents in LTC homes from 2013 to 2019. Residents' health resource utilization and quality-adjusted life-year (QALY) measures were extracted from multiple administrative databases. Six Markov states were modelled for post-antipsychotic progression representing PIUA, appropriate use of antipsychotic, complete withdrawal, and death. The primary outcome was the incremental cost per QALY gained.

Results A cohort of 35,669 residents was included in the primary analysis. The Clear initiative, over 10 years, was estimated to have an incremental cost-effectiveness ratio (ICER) of CA $\$ 26,055$ (2020 Canadian dollars) per QALY gained at an incremental cost of CA $\$ 5211$ per resident and a QALY gain of 0.20. In the subgroup analyses, our findings were even more favourable for Clear wave 2 (ICER of CA $\$ 24,447$ per QALY gained) and Clear wave 3 (ICER of CA \$25,933 per QALY gained). At a willingness-to-pay of CA $\$ 50,000$ per QALY gained, the probabilities of Clear waves 2 and 3 were $82 \%$ cost-effective. Conclusion This study demonstrated incremental costs and yielded favourable ICERs for Clear compared with the baseline. More research is needed to understand the level of support for individual care homes to sustain the Clear initiative in the long run.
\end{abstract}

\section{Introduction}

Antipsychotic medications are often prescribed to patients diagnosed with Alzheimer's disease, particularly for managing behavioural and psychological symptoms of dementia (BPSD), including aggression, delusion, and agitation [1, 2]. However, the use of antipsychotics in patients without a diagnosis of psychosis is classified as 'potentially inappropriate' $[3,4]$. Potentially inappropriate use of antipsychotics (PIUA) raises concerns about safety, quality of care, and costs, primarily due to the escalated demands on the healthcare system [5]. There is a strong body of scientific evidence surrounding the side effects of antipsychotics in the elderly, particularly those with dementia, including a sudden drop in

Asif Raza Khowaja

akhowaja@brocku.ca

Extended author information available on the last page of the article

\section{Key Points for Decision Makers}

The quality improvement programmes targeting appropriate utilization of antipsychotics in long-term care homes require a minimal financial investment.

The health-related quality of life (HRQoL) score was relatively higher in residents who had stopped taking potentially inappropriate antipsychotics than those who were consistently taking the antipsychotic medications. The HRQoL scores were poor (i.e., much lower) in residents with intermittent use of antipsychotics.

A person-centred approach for reducing potentially inappropriate use of antipsychotics appears to be cost effective. 
blood pressure, sedation, falls, fractures, stroke, and death $[6,7]$. Previously conducted studies also indicated a higher number of Emergency Department (ED) visits and prolonged inpatient hospitalization associated with PIUA $[8,9]$.

In Canada, over 419,000 people aged 65 years and older are living with dementia, and nearly 78,000 new dementia cases are diagnosed annually [10]. The prevalence of PIUA is much higher in the residents of long-term care (LTC) homes than in the general elderly population [11]. According to the Canadian Institute for Health Information (CIHI), the proportion of PIUA ranges from 14 to $40 \%$ in Canadian LTC homes, representing a considerable variation between provinces or regions [12]. For example, in 2018/19, the percentage of PIUA was as high as 39.8\% (95\% confidence interval [CI] 16.6-17.8) of LTC residents in the Labrador-Grenfell Health region versus $14 \%$ (95\% CI 13.2-15) of LTC residents in Edmonton Zone [12]. Such provincial/regional differences further indicate contextual challenges with respect to the facility size, staffing, administrative structure, and organizational culture. All of these factors are pivotal to designing robust quality improvement (QI) programmes in LTC homes.

Reducing PIUA is a critical aspect of QI in LTC. In a rapidly changing environment, particularly in the context of LTC, where a one-size-fits-all approach is not applicable, there are implications for shifting resources in person-centred care for patients diagnosed with dementia [13]. According to the Alzheimer Society of Canada, a person-centred approach allows care providers to work with residents, their families, and informal caregivers to understand the underlying causes of BPSD better. Additionally, this model of care focuses on building resident's strengths/abilities as well as periodic medication reviews for discontinuing or reducing antipsychotics [14]. One such QI initiative is the Call for Less Antipsychotics in Long-Term Care (Clear) homes in British Columbia (BC) [15]. The Clear initiative was launched in 2013 (wave 1), followed by Clear wave 2 in 2015 and Clear wave 3 in 2017. The focus of Clear wave 1 was geared towards inter-professional teams and shared learning, including four regional in-person workshops, creating a website for free access to resources, and online webinars [16]. Building on Clear wave 1, the second wave engaged clinicians and pharmacists in the person-centred approach. Examples of strategies implemented across care homes in wave 2 included using non-pharmacological measures to respond to residents' needs, establishing a medication review plan for residents on antipsychotic medications, and implementing best practices for prescribing antipsychotics appropriately [17]. The third wave further strengthened programme activities, updated online resources, and involved regional health authorities to sustain the Clear initiative. Since the inception of the Clear initiative, LTC homes have formed action-and-improvement teams and received support through shared resources, improvement coaching, mentorship, and opportunities to collectively learn and participate in local, regional, and provincial activities [15].

In the policy context, health system planners and administrators need to know the evidence about the cost-effectiveness to inform resource allocation decisions. This became more relevant towards the end of Clear wave 3 because no subsequent waves were anticipated, and the evidence surrounding the economic impact of Clear was lacking. Additionally, previously conducted programme evaluations of Clear waves broadly focused on the implementation aspects, such as percent reduction of PIUA, participation in webinars or workshops, and qualitative excerpts from residents about the perceived benefits $[16,17]$. The health-related quality of life (HRQoL) in residents, however, was not considered in the previous evaluations. The quality-adjusted life-years (QALY) is a generic measure of HRQoL that captures both the quality and the quantity of life lived and is commonly used in the economic evaluation of health interventions [18]. Thus, a comprehensive understanding of the Clear programme implementation costs and residents' quality of life is critical to fill the knowledge gap and further strengthen the policy argument for the spread, scale-up, and sustainability of the Clear initiative. This study aimed to estimate the costeffectiveness of the Clear initiative compared with the status quo (i.e., pre-Clear, baseline) in BC.

\section{Materials and Methods}

\subsection{Study Design and Perspective of Analysis}

This study utilized a model-based, cost-utility analysis using the guidelines of the Canadian Agency for Drugs and Technology (CADTH) and the Panel on Cost Effectiveness in Health and Medicine [19, 20]. All costs were calculated from the perspective of a single public payer (i.e., BC Ministry of Health).

\subsection{Target Population, Setting, and Costs of Health Resource Utilization (HRU)}

The resident-level data were obtained from the BC Ministry of Health for Continuing Care Reporting System (CCRS) for a cohort of residents identified as PIUA from the Residential Assessment Instrument (RAI) 2.0 from April 1, 2012, to March 31, 2019. A resident taking antipsychotics without a diagnosis of psychosis, excluding residents with delusions, hallucination, Huntington's chorea, schizophrenia, and end-of-life residents, was classified as potentially inappropriate [21]. The RAI assessments are completed by nurses every 3 months for every resident in the LTC home [22]. A unique study identification code for each resident from the 
RAI database was linked to four databases including the Discharge Abstract Database (DAD) [23], National Ambulatory Care Reporting System (NACRS) [24], PharmaNet (PhNet) [25], and Medical Services Plan (MSP) [26]. Key variables in these databases included residents' physical and health conditions, medications and services, visits by general practitioners, ED visits, hospital admissions, and discharge outcomes.

Descriptive analysis was performed to calculate the frequency and proportion of antipsychotic use, adverse events (i.e., falls and fractures), and health facility utilization. Using a case-mix-group (CMG) methodology, the resource intensity weight (RIW) was multiplied by the cost of standard hospital stay (for the period 2013-2018) to calculate the hospitalization cost per resident-admission [27]. For ambulatory care, standard costs of GP and ED were multiplied by the number of visits to calculate the cost per resident-occurrence [28]. The costs of antipsychotic medications were extracted from the MSP database [26]. All costs were adjusted using the Canadian Consumer Price Index for Health and are expressed in 2020 Canadian dollars (CA\$). Previously conducted economic studies indicated clustering effect on costs, care-seeking practices, and HRQoL in healthcare facilities within a larger health system [29, 30]. In the context of LTC, where some care homes tend to have a much older population, residents may have severe comorbidities compared with other care homes. Other factors such as bed capacity, public-versus-private ownership, geographical location, staffing ratio, etc., differ between care homes. We used a generalized linear mixed-effect model to estimate the mean cost per resident, accounting for age, sex, education (fixed variables), and clustering by LTC home (random variable).

\subsection{Programme-Related Costs}

Personnel time spent on Clear activities was determined through an online survey of care providers and administrative staff in LTC homes. The unit cost of personnel time (hourly wage) was calculated from the Health Employer Association of BC (HEABC) collective agreement for 2019-2022 [31]. On the programme side, Clear spending was determined from the institutional financial reports from 2013 to 2019. Major cost buckets included resource development, monitoring, supportive supervision, and fee for clinical leadership. The total cost was divided by the number of assessments to calculate the cost per resident-assessment.

\subsection{Health-Related Quality-Adjusted Life-Years (HRQOL)}

The RAI database captures resident-level information on socio-demographic characteristics, clinical conditions including comorbidities, daily living activities, vision, speech, hearing, cognition, pain, and depression [23]. A previous study mapped RAI variables to Health Utility Index (HUI) version 3 attributes and provided weighted utility scores using Canadian tariffs [32]. We used these utility weights for RAI variables and applied the following validated equation to calculate the HRQoL scores:

$$
\begin{aligned}
\text { HRQoL Score }= & 1.371\left(\text { Utility }_{\text {vision }} \times \text { Utility }_{\text {hearing }}\right. \\
& \times \text { Utility }_{\text {speech }} \times \text { Utility }_{\text {ambulation }} \\
& \times \text { Utility }_{\text {dexterity }} \times \text { Utility }_{\text {emotion }} \\
& \left.\times \text { Utility }_{\text {cognition }} \times \text { Utility }_{\text {pain }}\right)-0.37
\end{aligned}
$$

\subsection{Economic Model}

In our study, the Markov model was adopted (Moriarty et al. 2019) [33] because of its ability to model recurrent adverse events (i.e., falls leading to hip fracture), post-event healthcare-seeking (i.e., hospitalization and/or emergency admissions), and mortality, which is an ongoing risk over time. The Markov model was chosen to allow for time dependency, a particularly important consideration in the context of older people receiving PIUA in LTC homes. Six mutually exclusive health states were modelled, including (1) PIUA, 0 days, (2) PIUA 1-6 days, (3) PIUA 7 days, (4) Appropriate antipsychotic use, (5) Completely withdrawn from antipsychotics, and (6) Death (Fig. 1). Three PIUA states represent the frequency of antipsychotic drug use in the last 7 days from the assessment date, as reported in the RAI database. The RAI assessments are done at the time of admission in an LTC home (i.e., initial assessment) and are repeated on a quarterly basis (see electronic supplementary material [ESM], Supplementary Appendix Table S1: time-varying transition probabilities). All patients entered the model under PIUA states (i.e., PIUA $1-6$ days $=5 \%$, and PIUA 7 days $=95 \%$ ) and made transitions (to or from) or remained within any five states (except death state) in the subsequent quarterly cycles. There was a one-way transition to the death state, and those who died remained in the same state throughout the model cycles. The background annual mortality parameter was obtained from Statistics Canada for the BC population aged 78+ years [34]. Model structures were assessed for face validity by the research team (including geriatric clinicians), and models were cross-validated and compared to other published models concerning potentially inappropriate prescriptions [33]. We used double programming to validate the models. The model was first constructed in TreeAge Pro 2021 [35], and input parameters were assigned for all health states. Secondly, the model structures and corresponding values were exported in Microsoft Excel 2010 to detect structural or coding errors. The 
model structures programmed in TreeAge Pro and exported to Excel are included in Fig. 1.

\subsection{Cost-Utility Analysis}

Table 1 displays model input parameters derived from linked administrative databases, an online survey of care providers and administrators in LTC homes, a review of financial records, and grey literature. We analyzed costs and HRQoL in a 3-month (quarterly) cycle to calculate the incremental cost-effectiveness ratio (ICER). In the base-case analysis, we calculated ICERs for the Clear initiative compared with the status quo (pre-Clear baseline period). The ICER was calculated by dividing the difference in total costs (i.e., Clear versus status quo) by the difference in the outcomes (i.e., change in the HRQoL before and after Clear). Additionally, subgroup analyses were performed for each Clear wave 1,2 , and 3 compared with a common reference point of baseline. We calculated percent change in PIUA both within and between Clear waves compared with baseline (see Supplementary Appendix Table S1 in the ESM). Because each Clear wave's duration was roughly 2 years, we assumed a fixed average percent reduction in PIUA in the subsequent years. According to Statistics Canada, the life expectancy for adults aged 65 years in BC is 20.7 years [36]. In our study, the mean age of residents was 78.3 years (standard deviation \pm 10 ); therefore, we used a time horizon of 10 (i.e., to age 88 years) with a half-cycle correction. The longterm findings were extrapolated from 2-year QI data and so associated with some degree of uncertainty. Both costs and QALYs were discounted at $1.5 \%$ following the recent CADTH guidelines [19]. A sensitivity analysis was applied to test robustness with multiple scenarios by varying the effect size, Clear costs, and annual discount rate. In order to address the parameter uncertainties associated with the costs and outcome variables, the probabilistic analysis was applied using Monte Carlo simulations to draw values at random from 10,000 iterations. ICERs were reported using a standard willingness-to-pay (WTP) threshold of CA $\$ 50,000$ per QALY gain.

\section{Results}

\subsection{Baseline Characteristics}

A cohort of 35,669 residents from 312 LTC homes was included in the primary analysis. Small variations were observed in participants' characteristics in terms of clinical diagnosis, length of stay, and discharge outcomes in the post-Clear period compared with baseline. Of 35,669 residents, $53 \%$ of residents were over 85 years old $(n=18,762)$, $60 \%$ of residents were female $(n=21,613), 87 \%$ of residents were English speaking $(n=31,182)$, and $\sim 21 \%$ of residents attended high school $(n=7309)$. The leading medical diagnoses included hypertension ( $n=14,034 ; 39 \%)$, and Alzheimer disease $(n=5378,15 \%)$. Approximately $45 \%$ of residents stayed $<12$ months in the LTC home ( $n=15,918$ ), and over $50 \%$ of residents have died in this cohort $(n=22,604)$ (Table 2$)$.

\subsection{Resident-Level Outcomes}

A total of 381,434 resident RAI assessments were analyzed, translating into an average of $11 \mathrm{RAI}$ assessments per resident. Of 46,111 resident assessments in the baseline, the PIUA was reported in $58 \%$ of assessments $(n=26,563)$. The proportions of PIUA decreased to $52 \%$ in Clear wave $1(n=55,029$ out of 105,142$), 44 \%$ in Clear wave $2(n=49,290$ out of 111,395$)$, and $42 \%$ in Clear wave 3

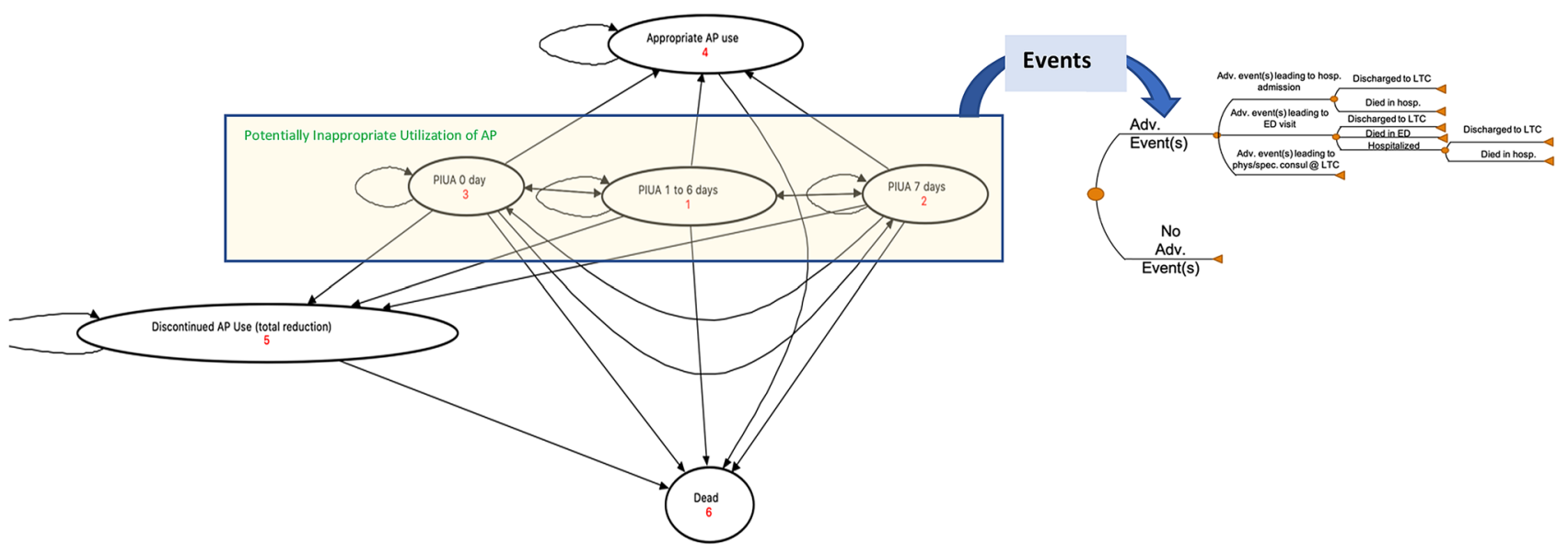

Fig. 1 Markov model. $A D V$ adverse, $A P$ antipsychotics, $E D$ emergency department, $L T C$ long-term care, PIUA potentially inappropriate use of antipsychotics 
Table 1 Model input parameters in the cost-effectiveness analysis

\begin{tabular}{|c|c|c|c|c|c|}
\hline Variable name & Value & Low & High & Distribution & Source \\
\hline \multicolumn{6}{|l|}{ Average costs per resident, in CA\$ } \\
\hline ED, per visit & 434 & 326 & 543 & Gamma & NACRS database BC \\
\hline MSP for ED, per visit & 86 & 65 & 108 & Gamma & MSP database BC \\
\hline Inpatient hospitalization, per admission & 13,747 & 10310 & 17184 & Gamma & DAD database BC \\
\hline MSP for an inpatient hospitalization, per admission & 93 & 70 & 116 & Gamma & MSP database BC \\
\hline MSP for care home consultation, per visit & 45 & 34 & 56 & Gamma & MSP database BC \\
\hline Antipsychotic medications, per claim & 13 & 10 & 16 & Gamma & PhNet database BC \\
\hline Onsite Clear implementation, per resident-assessment & 327 & 245 & 409 & Gamma & Clear-online survey \\
\hline $\begin{array}{l}\text { Clear (all waves combined) development and support, per resi- } \\
\text { dent-assessment }\end{array}$ & 187 & 140 & 234 & Gamma & Clear-review of financial report \\
\hline Clear wave 1 development and support, per resident-assessment & 272 & 204 & 340 & Gamma & Clear-review of financial report \\
\hline Clear wave 2 development and support, per resident-assessment & 119 & 89 & 149 & Gamma & Clear-review of financial report \\
\hline Clear wave 3 development and support, per resident-assessment & 229 & 172 & 286 & Gamma & Clear-review of financial report \\
\hline \multicolumn{6}{|l|}{ Adverse events per resident, probability } \\
\hline Hip fracture, baseline & 0.015 & 0.0135 & 0.0165 & Beta & RAI database BC \\
\hline Hip fracture, overall Clear (waves 1-3) & 0.014 & 0.0126 & 0.0154 & Beta & RAI database BC \\
\hline Hip fracture, Clear wave 1 & 0.014 & 0.0126 & 0.0154 & Beta & RAI database BC \\
\hline Hip fracture, Clear wave 2 & 0.014 & 0.0126 & 0.0154 & Beta & RAI database BC \\
\hline Hip fracture, Clear wave 3 & 0.013 & 0.0117 & 0.0143 & Beta & RAI database BC \\
\hline Inpatient hospital admission, baseline & 0.035 & 0.0315 & 0.0385 & Beta & RAI database BC \\
\hline Inpatient hospital admission, overall Clear (waves 1-3) & 0.034 & 0.0306 & 0.0374 & Beta & RAI database BC \\
\hline Inpatient hospital admission, Clear wave 1 & 0.035 & 0.0315 & 0.0385 & Beta & RAI database BC \\
\hline Inpatient hospital admission, Clear wave 2 & 0.035 & 0.0315 & 0.0385 & Beta & RAI database BC \\
\hline Inpatient hospital admission, Clear wave 3 & 0.033 & 0.0297 & 0.0363 & Beta & RAI database BC \\
\hline ED visit, baseline & 0.030 & 0.0270 & 0.0330 & Beta & RAI database BC \\
\hline ED visit, overall Clear (waves $1-3$ ) & 0.027 & 0.0243 & 0.0297 & Beta & RAI database BC \\
\hline ED visit, Clear wave 1 & 0.029 & 0.0261 & 0.0319 & Beta & RAI database BC \\
\hline ED visit, Clear wave 2 & 0.028 & 0.0252 & 0.0308 & Beta & RAI database BC \\
\hline ED visit, Clear wave 3 & 0.024 & 0.0216 & 0.0264 & Beta & RAI database $\mathrm{BC}$ \\
\hline Inpatient hospital admission from ED & 0.42 & 0.3780 & 0.4620 & Beta & RAI database BC \\
\hline Background mortality & 0.027 & 0.0230 & 0.0311 & Beta & Statistics Canada, BC province \\
\hline ED mortality & 0.001 & 0.0009 & 0.0011 & Beta & RAI database BC \\
\hline Inpatient hospital admission mortality & 0.00047 & 0.0004 & 0.0005 & Beta & RAI database BC \\
\hline \multicolumn{6}{|l|}{ Average potentially inappropriate use of antipsychotics per resident, $\%$} \\
\hline Baseline & 58 & 52 & 64 & Log Normal & RAI database BC \\
\hline Overall Clear (waves 1-3) & 46 & 41 & 51 & Log Normal & RAI database BC \\
\hline Clear wave 1 & 52 & 47 & 57 & Log Normal & RAI database BC \\
\hline Clear wave 2 & 44 & 40 & 48 & Log Normal & RAI database BC \\
\hline Clear wave 3 & 42 & 38 & 46 & Log Normal & RAI database BC \\
\hline 0 days, baseline & 30 & 28 & 33 & Log Normal & RAI database BC \\
\hline 0 days, overall Clear (waves $1-3$ ) & 40 & 36 & 44 & Log Normal & RAI database BC \\
\hline 0 days, Clear wave 1 & 37 & 33 & 41 & Log Normal & RAI database BC \\
\hline 0 days, Clear wave 2 & 42 & 38 & 46 & Log Normal & RAI database BC \\
\hline 0 days, Clear wave 3 & 41 & 37 & 45 & Log Normal & RAI database BC \\
\hline 1-6 days, baseline & 4 & 3.6 & 4.4 & Log Normal & RAI database BC \\
\hline 1-6 days, overall Clear (waves 1-3) & 3 & 2.7 & 3.3 & Log Normal & RAI database BC \\
\hline $1-6$ days, Clear wave 1 & 3 & 2.7 & 3.3 & Log Normal & RAI database BC \\
\hline $1-6$ days, Clear wave 2 & 3 & 2.7 & 3.3 & Log Normal & RAI database BC \\
\hline $1-6$ days, Clear wave 3 & 3 & 2.7 & 3.3 & Log Normal & RAI database BC \\
\hline 7 days, baseline & 66 & 59 & 73 & Log Normal & RAI database BC \\
\hline
\end{tabular}


Table 1 (continued)

\begin{tabular}{|c|c|c|c|c|c|}
\hline Variable name & Value & Low & High & Distribution & Source \\
\hline 7 days, overall Clear (waves $1-3$ ) & 57 & 51 & 63 & Log Normal & RAI database BC \\
\hline 7 days, Clear wave 1 & 60 & 54 & 66 & Log Normal & RAI database BC \\
\hline 7 days, Clear wave 2 & 55 & 50 & 61 & Log Normal & RAI database $\mathrm{BC}$ \\
\hline 7 days, Clear wave 3 & 56 & 50 & 62 & Log Normal & RAI database BC \\
\hline \multicolumn{6}{|c|}{ Average appropriate use of antipsychotics per resident, $\%$} \\
\hline Baseline and Clear waves & 15 & 13 & 17 & Beta & RAI database BC \\
\hline \multicolumn{6}{|c|}{ Effect size (total reduction in antipsychotics use), $\%$} \\
\hline Clear wave 1 & 9 & 8 & 10 & Beta & Calculated, RAI BC \\
\hline Clear wave 2 & 23 & 21 & 25 & Beta & Calculated, RAI BC \\
\hline Clear wave 3 & 27 & 24 & 31 & Beta & Calculated, RAI BC \\
\hline Average overall Clear (waves 1-3) & 20 & 18 & 22 & Beta & Calculated, RAI BC \\
\hline \multicolumn{6}{|c|}{ Health-related quality of life per resident on antipsychotics, utility score } \\
\hline 0 days & 0.859 & 0.7731 & 0.9449 & Beta & RAI database BC \\
\hline $1-6$ days & 0.843 & 0.7587 & 0.9273 & Beta & RAI database BC \\
\hline 7 days & 0.855 & 0.7695 & 0.9405 & Beta & RAI database BC \\
\hline \multicolumn{6}{|l|}{ Discounting, $\%$} \\
\hline Annual rate of discount & 1.5 & 0 & 5 & Beta & CADTH \\
\hline
\end{tabular}

$B C$ British Columbia, CADTH Canadian Agency for Drugs and Technology, DAD Discharge Abstract Database, ED emergency department, MSP Medical Services Plan, NACRS National Ambulatory Care Reporting System, PhNet Pharma Net, RAI Residential Assessment Instrument

( $n=50,544$ out of 119,786). Overall, a moderate percentage of residents (13-17\%) in PIUA states later transitioned into the appropriate antipsychotic state (i.e., diagnosed with psychosis) in the subsequent cycles. The proportion of residents on 0 days of PIUA was as high as $40 \%$ in Clear compared to baseline, where it was $30 \%$ (i.e., $10 \%$ reduction). Similarly, the frequency of PIUA was reduced in $41 \%$ of residents (1-6 days) in Clear compared with the baseline. Similarly, the proportion of residents on PIUA for 7 days declined to $57 \%$ in Clear compared with baseline, where it was $66 \%$ (see Supplementary Appendix Table S1 in the ESM). The HRQoL was marginally higher (i.e., mean score $0.859)$ in residents who stopped taking antipsychotics ( 0 days) compared with a mean score of 0.854 in residents who were taking antipsychotic medications 7 days a week. The HRQoL was lower (mean score 0.843) in residents taking antipsychotic medication for 1-6 days per week (Fig. 2). Hospital admission was $3.5 \%$ ( $n=921$ out of 26,563 ) in the baseline compared with $3.3 \%$ ( $n=1655$ out of 50,544$)$ in Clear wave 3 . Similarly, the emergency visits declined to $2.4 \%(n=1234$ out of 50,544) in Clear wave 3 compared with $3 \%$ for baseline ( $n=788$ out of 26,563$)$. The proportion of residents reported with hip fractures, other fractures, and the use of trunk restraint declined minimally in Clear waves 1, 2, and 3 compared with baseline (Table 3 ).

\subsection{Clear Costs}

Table 4 reports on the Clear-related costs borne by the $\mathrm{BC}$ Patient Safety and Quality Council (BCPSQC). Approximately CA $\$ 2.2 \mathrm{M}$ was spent on the Clear initiative in $\mathrm{BC}$ (all waves inclusive). Clear wave 1 represents a total of CA $\$ 819,218$ ( CA $\$ 272$ per resident assessment) compared with CA $\$ 669,199$ ( CA $\$ 229$ per resident assessment) in Clear wave 3. The costs were lower ( CA $\$ 119$ per resident assessment) in Clear wave 2 due to a large cohort of residents using antipsychotics. Overall, the main spending buckets were personnel compensation, including benefits (72\%), and professional clinical leadership fees (11\%).

\subsection{Model-Based Cost-Effectiveness Analysis}

The Clear initiative (i.e., all waves combined), over 10 years, was estimated to have an incremental cost of CA\$5211 and incremental QALY of 0.20 per resident (i.e., the ICER was CA $\$ 26,055$ per QALY gained). In the sub-group analysis, Clear waves 2 and 3 yielded much lower ICERs (i.e., the ICERs were CA $\$ 24,447$ per QALY gained in wave 2 and CA $\$ 25,933$ per QALY gained in wave 3) compared with the baseline over 10 years (Table 5). When the percentage of inappropriate antipsychotic use was decreased from 46 to $41 \%$ (i.e., a $10 \%$ reduction), the ICER was estimated to be lower (i.e., CA $\$ 22,840$ per QALY gain) in the overall Clear strategy compared with baseline. Similarly, when the average cost of Clear implementation by sites was assumed 
Table 2 Participants' characteristics, clinical diagnosis, length of stay and discharge

\begin{tabular}{|c|c|c|c|c|c|}
\hline \multirow[t]{2}{*}{ Variables } & \multirow[t]{2}{*}{ Baseline } & \multicolumn{3}{|l|}{ Clear waves } & \multirow[t]{2}{*}{ Total } \\
\hline & & 1 & 2 & 3 & \\
\hline Number of residents & 13,700 & 7728 & 7155 & 7086 & 35,669 \\
\hline \multicolumn{6}{|l|}{ Gender } \\
\hline Female, $n(\%)$ & $8866(64.7)$ & $4636(60.0)$ & 4109 (57.4) & $4002(56.5)$ & $21,613(60.6)$ \\
\hline \multicolumn{6}{|l|}{ Age group (in years), $n(\%)$} \\
\hline $65-74$ & $1602(11.7)$ & $977(12.6)$ & $941(13.2)$ & $997(14.1)$ & $4517(12.7)$ \\
\hline $75-84$ & $4522(33.0)$ & $2771(35.9)$ & $2578(36.0)$ & $2519(35.5)$ & $12,390(34.7)$ \\
\hline $85+$ & $7576(55.3)$ & $3980(51.5)$ & $3636(50.8)$ & $3570(50.4)$ & $18,762(52.6)$ \\
\hline \multicolumn{6}{|l|}{ Language } \\
\hline English & $12,022(87.8)$ & $6799(88.0)$ & $6165(86.2)$ & $6195(87.4)$ & $31,182(87.4)$ \\
\hline Panjabi & $104(0.8)$ & $73(0.9)$ & $88(1.2)$ & $102(1.4)$ & $367(1.0)$ \\
\hline Chinese & $27(0.2)$ & $215(2.8)$ & $169(2.4)$ & $145(2.1)$ & 557 (1.6) \\
\hline Italian & $138(1.0)$ & $90(1.2)$ & $88(1.2)$ & $86(1.2)$ & $402(1.1)$ \\
\hline Others & $1409(10.3)$ & $550(7.1)$ & $645(9.0)$ & $558(7.9)$ & $3161(8.9)$ \\
\hline \multicolumn{6}{|l|}{ Education } \\
\hline No schooling & $230(1.7)$ & $123(1.6)$ & $111(1.6)$ & $92(1.3)$ & $557(1.6)$ \\
\hline 8th grade or less & $2332(17.0)$ & $1260(16.3)$ & $1034(14.4)$ & $1001(14.1)$ & $5627(15.8)$ \\
\hline 9 th to 11 th grade & $2220(16.2)$ & $1211(15.7)$ & $1114(15.6)$ & $1128(15.9)$ & $5673(15.9)$ \\
\hline High school & $2723(19.9)$ & $1576(20.4)$ & 1565 (21.9) & $1445(20.4)$ & $7309(20.5)$ \\
\hline Technical or trade school & $975(7.1)$ & $653(8.4)$ & $577(8.1)$ & $580(8.2)$ & $2785(7.8)$ \\
\hline Some college & $936(6.8)$ & $573(7.4)$ & $548(7.7)$ & $608(8.6)$ & $2664(7.5)$ \\
\hline Bachelor's degree & $727(5.3)$ & $511(6.6)$ & $475(6.6)$ & $533(7.5)$ & $2245(6.3)$ \\
\hline Graduate degree & $391(2.9)$ & $230(3.0)$ & $214(3.0)$ & $244(3.4)$ & $1080(3.0)$ \\
\hline Unknown & $3166(23.1)$ & $1591(20.6)$ & $1518(21.2)$ & $1455(20.5)$ & $7729(21.7)$ \\
\hline \multicolumn{6}{|l|}{ Selected medical diagnosis } \\
\hline Hypertension & $2971(21.7)$ & $3772(48.8)$ & $3635(50.8)$ & $3655(51.6)$ & $14,034(39.3)$ \\
\hline Asthma & $156(1.1)$ & $187(2.4)$ & $160(2.2)$ & $184(2.6)$ & $688(1.9)$ \\
\hline Alzheimer disease & $1191(8.7)$ & $1451(18.8)$ & $1373(19.2)$ & $1363(19.2)$ & $5378(15.0)$ \\
\hline Kidney disease & $525(3.8)$ & $730(9.5)$ & $775(10.8)$ & $823(11.6)$ & $2854(8.0)$ \\
\hline \multicolumn{6}{|l|}{ Activity of daily living } \\
\hline Independent & $6743(49.2)$ & $4040(52.3)$ & $3685(51.5)$ & $3655(51.6)$ & $18,124(50.8)$ \\
\hline Supervision & $1447(10.6)$ & 1066 (13.8) & $1106(15.5)$ & $1026(14.5)$ & $4644(13.0)$ \\
\hline Limited assistance & $1374(10.0)$ & $831(10.8)$ & $697(9.7)$ & $683(9.6)$ & $3584(10.0)$ \\
\hline Extensive assistance & $1024(7.5)$ & $587(7.6)$ & $580(8.1)$ & $547(7.7)$ & $2739(7.7)$ \\
\hline Total dependence & $2582(18.8)$ & $987(12.8)$ & $885(12.4)$ & $943(13.3)$ & $5397(15.1)$ \\
\hline Activity did not occur & $530(3.9)$ & $216(2.8)$ & $203(2.8)$ & $232(3.3)$ & $1181(3.3)$ \\
\hline \multicolumn{6}{|l|}{ Self performance_eating } \\
\hline Independent & $6823(49.8)$ & $4206(54.4)$ & $3824(53.4)$ & $3749(52.9)$ & $18,602(52.1)$ \\
\hline Supervision & $3318(24.2)$ & $2176(28.2)$ & $2101(29.4)$ & $2133(30.1)$ & $9728(27.3)$ \\
\hline Limited assistance & $1307(9.5)$ & $616(8.0)$ & 547 (7.6) & $524(7.4)$ & $2993(8.4)$ \\
\hline Extensive assistance & $928(6.8)$ & $362(4.7)$ & $317(4.4)$ & $328(4.6)$ & $1936(5.4)$ \\
\hline Total dependence & $1315(9.6)$ & $361(4.7)$ & $365(5.1)$ & $346(4.9)$ & $2388(6.7)$ \\
\hline Activity did not occur & $9(0.1)$ & $7(0.1)$ & $1(0.0)$ & $6(0.1)$ & $23(0.1)$ \\
\hline \multicolumn{6}{|l|}{ Facility length of stay, months } \\
\hline$<12$ & $2557(18.7)$ & $3391(43.9)$ & $3992(55.8)$ & $5978(84.4)$ & $15,918(44.5)$ \\
\hline $12-24$ & $1492(10.9)$ & $1455(18.8)$ & $1497(20.9)$ & $949(13.4)$ & $5393(15.1)$ \\
\hline$>24$ & $9651(70.4)$ & $2882(37.3)$ & $1666(23.3)$ & $159(2.2)$ & $14,358(40.4)$ \\
\hline \multicolumn{6}{|l|}{ Reasons for discharge } \\
\hline Deceased & $11,116(81.1)$ & $5235(67.7)$ & $4021(56.2)$ & $2232(31.5)$ & $22,604(63.4)$ \\
\hline
\end{tabular}


Table 2 (continued)

\begin{tabular}{|c|c|c|c|c|c|}
\hline \multirow[t]{2}{*}{ Variables } & \multirow[t]{2}{*}{ Baseline } & \multicolumn{3}{|l|}{ Clear waves } & \multirow[t]{2}{*}{ Total } \\
\hline & & 1 & 2 & 3 & \\
\hline Hospital—inpatient acute care & $260(1.9)$ & $167(2.2)$ & $196(2.7)$ & $150(2.1)$ & $774(2.2)$ \\
\hline Hospital —inpatient psychiatric care & $25(0.2)$ & $38(0.5)$ & $38(0.5)$ & $37(0.5)$ & $138(0.4)$ \\
\hline Hospital-inpatient continuing care & $16(0.1)$ & $9(0.1)$ & $12(0.2)$ & $17(0.2)$ & $54(0.2)$ \\
\hline Hospital-inpatient rehabilitation & $10(0.1)$ & $4(0.05)$ & $1(0.01)$ & $7(0.1)$ & $22(0.1)$ \\
\hline Hospital—ambulatory health service & $11(0.1)$ & $2(0.02)$ & $2(0.03)$ & $4(0.1)$ & $19(0.1)$ \\
\hline Residential care-24-h nursing care & $493(3.6)$ & $429(5.5)$ & $539(7.5)$ & $734(10.4)$ & $2195(6.1)$ \\
\hline Residential care-board and care & $91(0.7)$ & $94(1.2)$ & $104(1.5)$ & $138(1.9)$ & $427(1.2)$ \\
\hline Private home-without care & $96(0.7)$ & $74(1.0)$ & $71(1.0)$ & $61(0.9)$ & $303(0.8)$ \\
\hline Private home-with care & $60(0.4)$ & $58(0.7)$ & $31(0.4)$ & $30(0.4)$ & $179(0.5)$ \\
\hline Other or unknown discharge disposition & $46(0.3)$ & $28(0.4)$ & $40(0.6)$ & $43(0.6)$ & $157(0.4)$ \\
\hline Still in LTC & $1475(10.8)$ & $1592(20.6)$ & $2099(29.3)$ & $3633(51.3)$ & $8797(24.6)$ \\
\hline
\end{tabular}

LTC long-term care

Fig. 2 Health-related quality of life

Table 3 Adverse events and health resource utilization

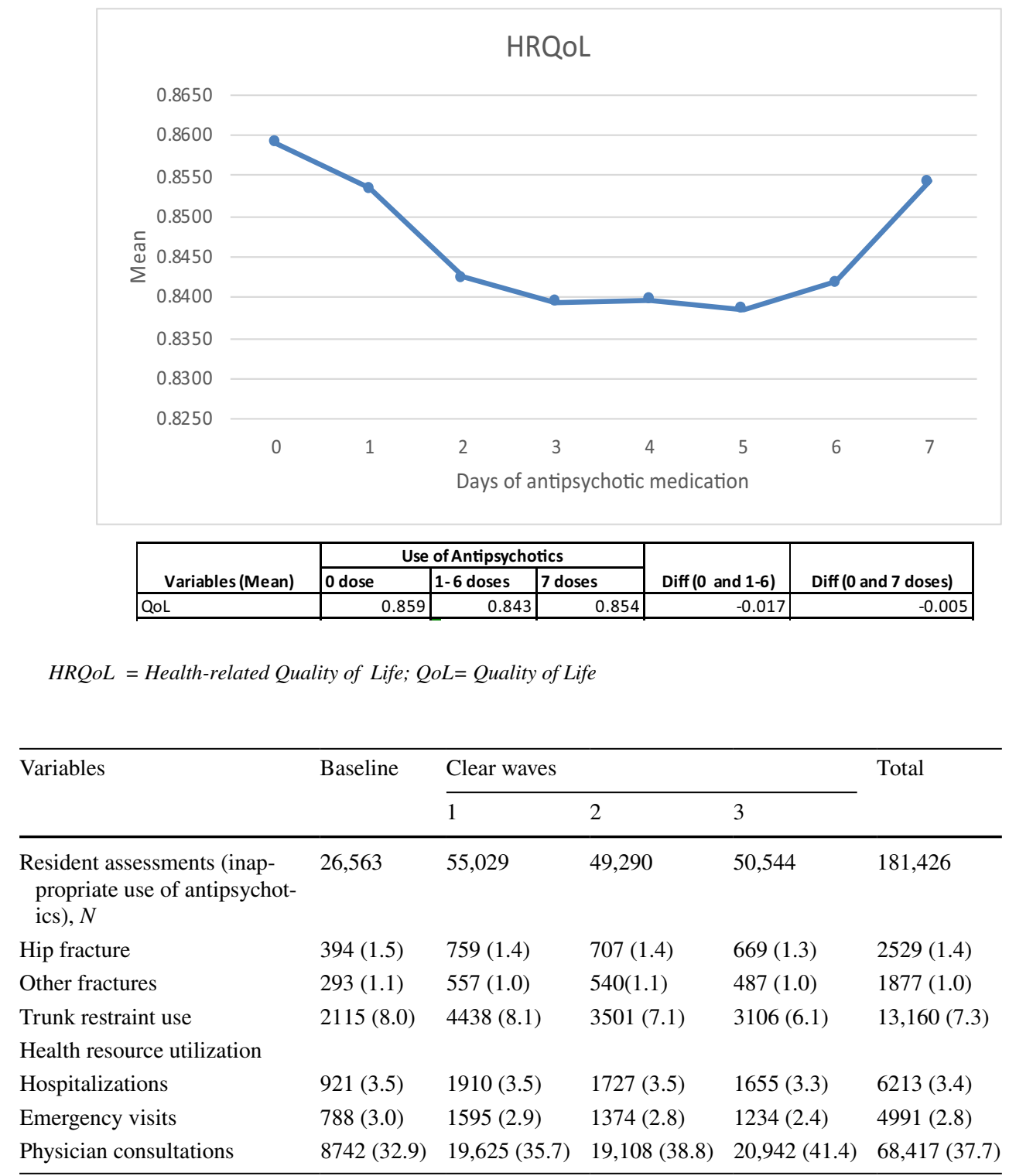


to increase from $\mathrm{CA} \$ 327$ to $\mathrm{CA} \$ 409$ per resident (i.e., a $25 \%$ inflation), the ICER was estimated to be higher (i.e., CA $\$ 29,872$ per QALY gain). In other scenarios, when changes were made in the discount rate and Clear programme costs borne by the BCPSQC, minimal variations were observed in the ICERs under the strategy of overall Clear waves (1-3) compared with the baseline (Fig. 3). In the probabilistic analysis, ICERs were favourable for Clear waves 2 and 3, and overall. For example, the costeffectiveness plane revealed most ICER points under the WTP threshold of CA $\$ 50,000$ per QALY gained (Fig. 4). In the cost-effectiveness acceptability curve, the probabilities of Clear waves 2 and 3 were $82 \%$ cost effective under the CA $\$ 50 \mathrm{~K}$ WTP threshold. However, Clear wave 1 was approximately $36 \%$ cost effective at the same threshold (Fig. 5).

\section{Discussion}

This study demonstrated a significant reduction in the PIUA in LTC homes in the post-Clear period compared with the baseline. The financial analysis indicated minimal costs of the Clear initiative from the perspective of the public payer in BC. The subgroup analyses, however, revealed variation in the ICERs between Clear waves. For example, Clear wave 1 had a relatively higher ICER of CA $\$ 96,011$ per QALY gained compared with Clear wave 2 (ICER of CA \$24,447). In understanding these differences, the first explanation may be that programme-level spending in Clear wave 1 was much higher at CA $\$ 819,218$ (38\% out of total Clear spending of CA $\$ 2,177,555)$. Clear wave 1 was more resource intensive in terms of setting up data flow and communication pathways, developing resources including production and graphic design, initial planning, and engagement meetings with care homes than the subsequent waves. Second, it may be that the Clear initiative was in the early stage of the rollout; therefore, it did not make a large impact on PIUA,

Table 4 Clear initiative yearly spending 2013-2019

\begin{tabular}{|c|c|c|c|c|c|c|c|}
\hline \multirow[t]{2}{*}{ Budget line } & \multicolumn{2}{|l|}{ Wave 1} & \multicolumn{2}{|l|}{ Wave 2} & \multicolumn{2}{|l|}{ Wave 3} & \multirow{2}{*}{ Total } \\
\hline & 2013-2014 & 2014-2015 & 2015-2016 & 2016-2017 & 2017-2018 & 2018-2019 & \\
\hline Data \& communications & 181.83 & - & - & - & - & - & 181.83 \\
\hline Workshop fees & $27,945.82$ & 21486.59 & 20219.03 & 17533.91 & 10207.69 & 6279.38 & $103,672.42$ \\
\hline Conference fees (staff) & - & 160.00 & - & 1882.03 & 24.29 & - & 2066.32 \\
\hline Subscription fees & - & - & - & 17.53 & - & - & 17.53 \\
\hline Production & $12,200.49$ & 2053.12 & 1318.67 & 412.60 & 7069.53 & - & $23,054.41$ \\
\hline Graphic design & 3352.63 & 3587.50 & 1544.06 & - & - & - & 8484.19 \\
\hline Sundry other & - & 62.26 & - & - & - & - & 62.26 \\
\hline Sponsorships & 2485.94 & - & - & - & - & - & 2485.94 \\
\hline Meeting expense & 3250.53 & - & - & 1090.80 & 615.03 & 323.85 & 5280.21 \\
\hline Postage & 480.72 & - & 1074.43 & 40.01 & 426.10 & 51.26 & 2072.52 \\
\hline Delivery and courier & - & - & 158.78 & 313.64 & 387.85 & 155.07 & 1015.34 \\
\hline Consultants general & - & - & - & 31129.87 & 27140.56 & - & $58,270.43$ \\
\hline Prof fees (non-physicians) & $11,495.37$ & 60198.24 & 22796.92 & 35709.16 & 39367.62 & 67266.05 & $236,833.36$ \\
\hline Prof fees (physicians) & $38,059.13$ & 36100.33 & 7205.28 & 2150.17 & 631.43 & 2866.54 & $87,012.88$ \\
\hline Prof fees (travel) & 488.87 & 1052.69 & 1825.86 & 1007.58 & 3312.90 & 562.96 & 8250.86 \\
\hline Honorariums & - & - & - & - & - & 250.00 & 250.00 \\
\hline Board local travel & 59.15 & - & - & - & - & - & 59.15 \\
\hline Staff local & 224.40 & 280.98 & 373.30 & 61.66 & 561.78 & 154.39 & 1656.51 \\
\hline Staff provincial & 9112.21 & 8794.91 & 5297.30 & 14081.05 & 17028.15 & 12335.81 & $66,649.43$ \\
\hline Staff out of province & 518.33 & 1293.03 & - & 1313.39 & 92.87 & - & 3217.62 \\
\hline General office supplies & - & - & - & 70.56 & 190.15 & 15.54 & 276.25 \\
\hline $\begin{array}{l}\text { Personnel compensation, } \\
\text { including benefits }\end{array}$ & $287,146.64$ & $287,146.64$ & $260,254.65$ & $260,254.65$ & $275,845.67$ & $196,036.97$ & $1,566,685.22$ \\
\hline Total & $397,002.06$ & $422,216.29$ & $322,068.28$ & $367,068.61$ & $382,901.62$ & $286,297.82$ & $2,177,554.68$ \\
\hline
\end{tabular}


Table 5 Cost effectiveness of Clear initiative in $\mathrm{BC}$ over a 10 -year time horizon

\begin{tabular}{lcllll}
\hline Strategy & $\begin{array}{l}\text { Discounted } \\
\text { cost, CA\$ }\end{array}$ & $\begin{array}{l}\text { Incremental } \\
\text { cost, CA\$ }\end{array}$ & $\begin{array}{l}\text { Discounted } \\
\text { QALYs }\end{array}$ & $\begin{array}{l}\text { Incremental } \\
\text { QALYs }\end{array}$ & $\begin{array}{l}\text { ICER (Incr. Cost/ } \\
\text { Incr. QALYs), } \\
\text { CA\$ }\end{array}$ \\
\hline All referencing common baseline & & & & \\
Baseline & 943 & - & 4.02 & - & - \\
Clear wave 1 & 9584 & 8641 & 4.11 & 0.09 & 96,011 \\
Clear wave 2 & 5588 & 4645 & 4.21 & 0.19 & 24,447 \\
Clear wave 3 & 6389 & 5446 & 4.23 & 0.21 & 25,933 \\
Overall (waves 1-3) & 6154 & 5211 & 4.22 & 0.20 & 26,055 \\
\hline
\end{tabular}

$B C$ British Columbia, $C A \$$ Canadian dollars, $Q A L Y s$ quality-adjusted life-years

\section{Tornado Diagram - ICER Baseline vs. Clear Overallwaves}

Onsite cost of Clear implementation by care providers $( \pm 25 \%$

Inappropriate antipsychotic use $(+10 \%$

Clear costs bome by BCPSQC $( \pm 25 \%)$

Discount rate $(0,5 \%)$

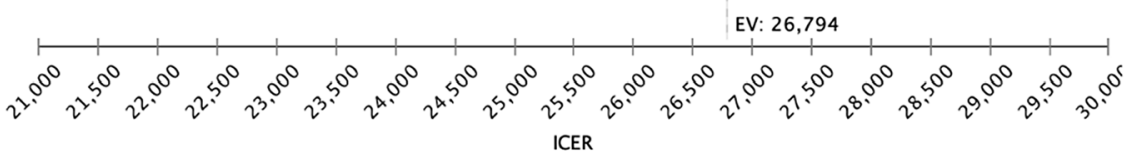

Fig. 3 Tornado diagram of one-way sensitivity analysis. The blue bars represent scenarios in which ICER is decreased. The red bars represent scenarios in which ICER is increased. BCPSQC British

translating into lower QALY gains of 0.09 in Clear wave 1 compared with baseline. Time to behaviour change is also important to consider, as the current prescribing practices within some LTC homes may have prevented any changes in antipsychotic use during Clear wave 1 . In addition, staff may not have seen any changes in the residents' activities of daily living and/or agitation levels in the early phase of the Clear initiative. Our findings are corroborated with a previously conducted trial-based cost-effectiveness analysis of PARO (a therapeutic robotic seal) to reduce agitation and medication use in residents identified with dementia. In that study, investigators found higher costs and no significant reduction in medication use and resident agitation over a 10-week intervention period [37]. Similarly, other studies involving a short duration ( $\leq 1$-year period) also found higher costs and no (or even negative) benefits of QI interventions $[38,39]$. Thus, our study findings, particularly that ICERs decreased in the subsequent Clear waves, put
Columbia Patient Safety and Quality Council, Clear Call for Less Antipsychotics in Long-Term Care, EV expected value, ICER incremental cost-effectiveness ratio

forward a strong advocacy argument for a longer duration of QI interventions in LTC.

As many provinces encourage less antipsychotic use in LTC, it is argued that there may be a shift/increase in other medications (e.g., benzodiazepines as needed) commonly used for sedation. Unfortunately, this cannot be fully determined as the current study analyzed secondary data (i.e., Pharmanet database) only for antipsychotic medication claims. More research is needed to evaluate the unintended consequences of antipsychotics reduction in LTC. Our findings illustrate the technical efficiency of a province-wide QI initiative that aimed to support a large number of LTC homes to provide person-centred care for residents with BPSD [40]. Nonetheless, promoting non-pharmacological interventions and periodic medication reviews for all LTC residents may be challenging in small care homes because of limited staffing capacity and resources [41]. This leads to a broader policy question whereby some care homes would require more support for implementing QI strategies and 

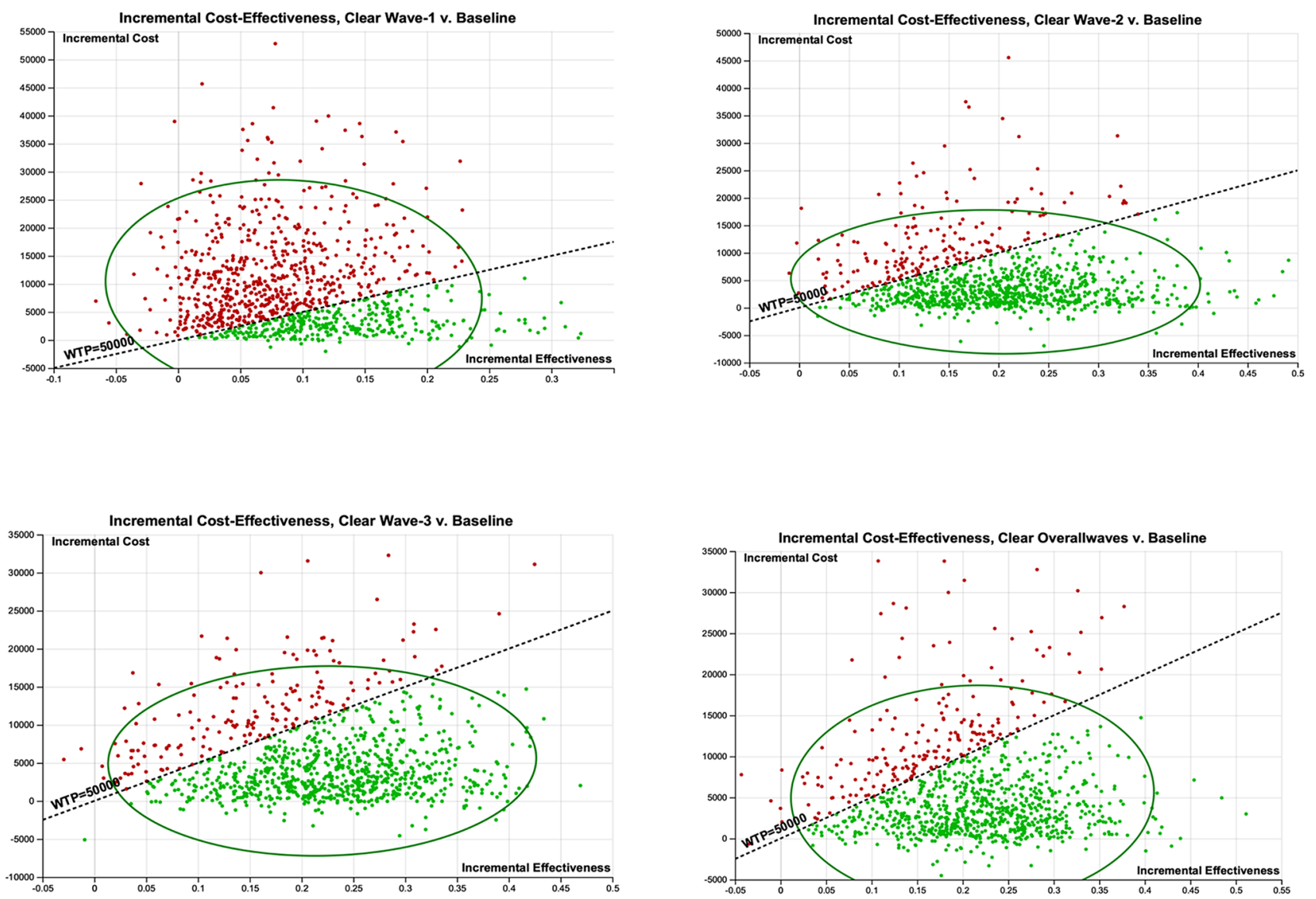

Fig. 4 Cost-effectiveness plane. The green dots represent scenarios in which ICER is below the CA $\$ 50 \mathrm{~K}$ threshold. The red dots represent scenarios in which ICER is above the CA $\$ 50 \mathrm{~K}$ threshold

Fig. 5 Cost-effectiveness acceptability curve

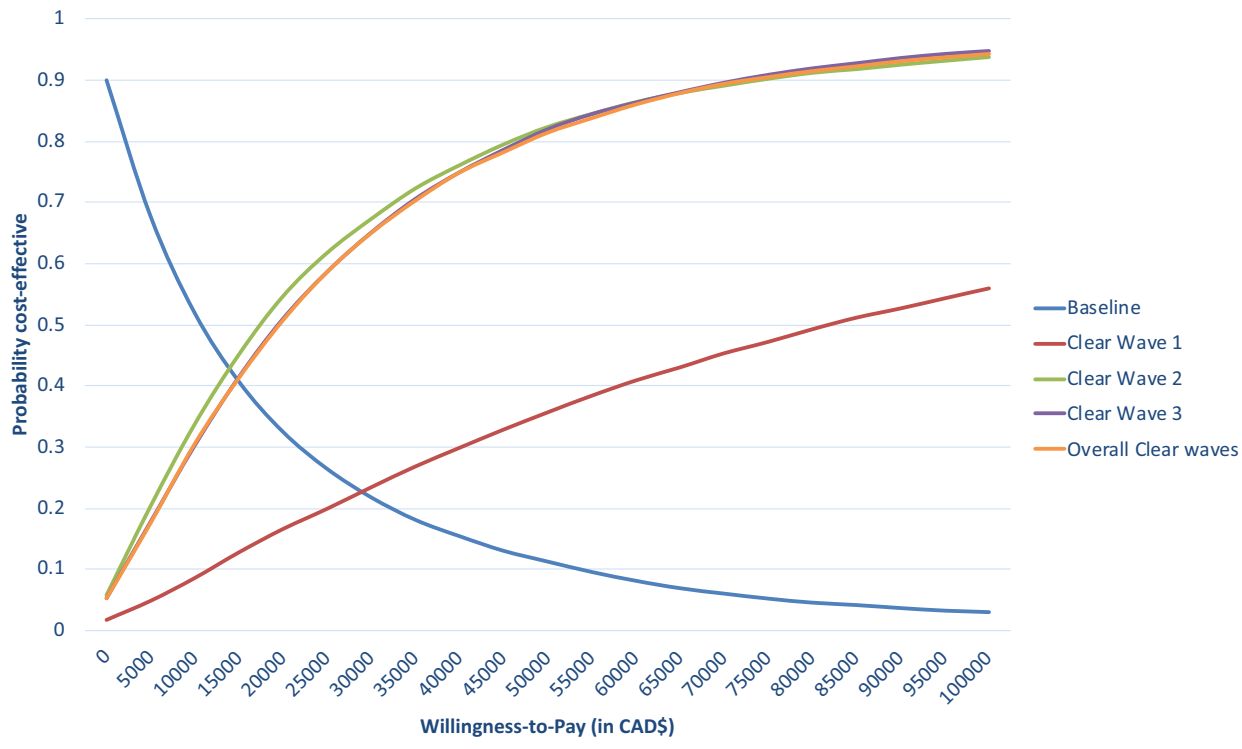


perhaps organizational incentive to sustain QI initiatives in the long run [42]. For example, regional health authorities might provide an enhanced institutional budget conditional on participating in such province-wide QI initiatives. Questions about the type and extent of support required by individual care homes could be further explored through implementation science research embedded in the planning phase of QI initiatives.

Despite other similar antipsychotic reduction initiatives in LTC across Canada, the Clear initiative is unique for its scope and scale. Examples of other initiatives in Canada include (1) the Alberta Appropriate Utilization of Antipsychotics (AUA) initiative [43] and (2) the pan-Canadian AUA collaborative initiative led by the Canadian Foundation for Healthcare Improvement in Prince Edward Island, New Brunswick, and Ontario [44]. Notably, many of these QI initiatives have been active for years and focused much less on non-technical skills. However, the Clear initiative emphasizes non-technical skills, including leadership, decision-making, situation awareness, communication, and teamwork, which are essential for team culture. By implementing a broader QI framework, the Clear initiative improved residents' quality of life, reduced HRU costs and ensured sustainability and ongoing engagement.

\subsection{Strengths and Limitations}

To the best of our knowledge, this is the first comprehensive cost-utility analysis of the QI initiative aiming to reduce PIUA in LTC in Canada. A large resident-level data pool from multiple administrative sources is a key strength of this study. This study used a provincial perspective, so residents who did not participate in the Clear initiative were also included in the cohort. Because data were encrypted, we could not stratify our analysis based on participation, geographical location, rurality, care home size determined by bed capacity, and type of funding received from health authority versus privately operated - this is a limitation of the secondary data analysis. Unlike the interventional studies in which the causal relationship (i.e., the effect of drug therapy or procedure on the HRQoL) can be quantitatively established, QI strategies predominately rely on soft indicators of change (time trends) and/or qualitative (anecdotal) experiences of change agents. We recognize the limitation of the pre-and-post study design and that many other factors (e.g., changes in policy, care home practices, attrition of care providers, etc.) were challenging to evaluate as part of this study. Moreover, the out-of-pocket costs and time/productivity losses (i.e., opportunity costs) could significantly burden residents' families. For example, residents who are awake (i.e., no longer chemically restrained with the PIUA) may require additional family/caregiver involvement in daily living activities. However, we did not find significant differences in residents' hospitalization rates in the Clear waves compared with the baseline (Table 3 ). Since we analyzed secondary data, evaluating the burden of indirect costs to family/caregivers was beyond this study's scope. Therefore, our study reports the Clear initiative's cost effectiveness using a less encompassing public-payer perspective, and more research is needed to evaluate societal costs.

\section{Conclusions}

Our findings show that the Clear initiative in BC, compared with the status quo (baseline), appears to be cost-effective in improving residents' quality of life. The person-centred approach, as part of the Clear initiative, suggested that care providers were able to engage with residents and their family members in the decision-making process of reducing PIUA, and in many cases, helped residents through various non-therapeutic strategies to achieve this goal. This study also highlights financial implications, albeit minimal, associated with an incremental cost for implementing Clear-related tasks for care homes. More research is needed on how small sites, which may have limited resources, could be supported by regional health authorities to sustain this initiative in the long run.

Supplementary Information The online version contains supplementary material available at https://doi.org/10.1007/s41669-021-00267-6.

Acknowledgements We thank BCPSQC staff (ASI team, HSI team and Clear Clinical faculty) for ongoing support, data request submission, office space, and analytical software access. We are grateful to the administrative staff and care providers in LTC homes for their time and online survey participation. We thank Eric Young for helping with the data access and Joelle DesChatelets for proof editing the manuscript.

\section{Declarations}

Funding This review was undertaken as part of the postdoctoral fellowship work jointly funded by the Canadian Institutes of Health Research (CIHR), Institute of Health Services and Policy Research (IHSPR), the Michael Smith Foundation for Health Research (MSFHR) and British Columbia Patient Safety \& Quality Council (BCPSQC).

Competing interests The authors declare that they have no competing interests.

Ethics approval This study was approved by the IRB of the University of British Columbia (H18-03581).

Consent to participate Not applicable.

Consent for publication Not applicable.

Availability of data and materials All data and materials will be provided upon request.

Code availability Not applicable. 
Author contributions $\mathrm{AK}, \mathrm{CK}$, and $\mathrm{CM}$ conceived the idea of the study. AK designed the model, undertook the literature review and wrote the first draft of the manuscript. All authors contributed to the subsequent redrafting of the manuscript and approved the final version.

Open Access This article is licensed under a Creative Commons Attribution-NonCommercial 4.0 International License, which permits any non-commercial use, sharing, adaptation, distribution and reproduction in any medium or format, as long as you give appropriate credit to the original author(s) and the source, provide a link to the Creative Commons licence, and indicate if changes were made. The images or other third party material in this article are included in the article's Creative Commons licence, unless indicated otherwise in a credit line to the material. If material is not included in the article's Creative Commons licence and your intended use is not permitted by statutory regulation or exceeds the permitted use, you will need to obtain permission directly from the copyright holder. To view a copy of this licence, visit http://creativecommons.org/licenses/by-nc/4.0/.

\section{References}

1. Ballard C, Creese B, Corbett A, Aarsland D. Atypical antipsychotics for the treatment of behavioral and psychological symptoms in dementia, with a particular focus on longer term outcomes and mortality. Expert Opin Drug Saf. 2011;10(1):35-43.

2. Parsons C, Johnston S, Mathie E, Baron N, Machen I, Amador S, Goodman C. Potentially inappropriate prescribing in older people with dementia in care homes. Drugs Aging. 2012;29(2):143-55.

3. Rios S, Perlman CM, Costa A, Heckman G, Hirdes JP, Mitchell L. Antipsychotics and dementia in Canada: a retrospective cross-sectional study of four health sectors. BMC Geriatr. 2017;17(1):244.

4. Hernández R, Gómez de Segura A, Medrano J. Potentially inappropriate medication in elderly. Psychopharmacol Issues Geriatrics Bentham Sci. 2015;2015:65-96.

5. Pasina L, Djade CD, Tettamanti M, Franchi C, Salerno F, Corrao S, Marengoni A, Marcucci M, Mannucci PM, Nobili A, REPOSI Investigators. Prevalence of potentially inappropriate medications and risk of adverse clinical outcome in a cohort of hospitalized elderly patients: results from the REPOSI Study. J Clin Pharm Therapeutics 2014;39(5):511-5.

6. Champoux N, Monette J, Monette M, du Fort GG, Wolfson C, Le Cruguel JP. Use of neuroleptics: study of institutionalized elderly people in Montreal, Que. Can Family Phys. 2005;51(5):696-7.

7. Hill KD, Wee R. Psychotropic drug-induced falls in older people. Drugs Aging. 2012;29(1):15-30.

8. Rochon PA, Normand SL, Gomes T, Gill SS, Anderson GM, Melo M, Sykora K, Lipscombe L, Bell CM, Gurwitz JH. Antipsychotic therapy and short-term serious events in older adults with dementia. Arch Intern Med. 2008;168(10):1090-6.

9. Frankenthal D, Lerman Y, Lerman Y. The impact of hospitalization on potentially inappropriate prescribing in an acute medical geriatric division. Int J Clin Pharm. 2015;37(1):60-7.

10. Government of Canada. Dementia in Canada, including Alzheimer's disease. 2017. https://www.canada.ca/en/public-health/servi ces/publications/diseases-conditions/dementia-highlights-canad ian-chronic-disease-surveillance.html. Accessed 30 Nov 2020.

11. Vida S, Monette J, Wilchesky M, Monette M, Friedman R, Nguyen A, Dastoor D, Cristache G, Sourial N, Tremblay L, Gore B. A long-term care center interdisciplinary education program for antipsychotic use in dementia: program update five years later. Int Psychogeriatr. 2012;24(4):599.

12. Canadian Institute for Health Information. Potentially inappropriate use of antipsychotics in long-term care. 2020. https://yourh ealthsystem.cihi.ca/hsp/inbrief?lang=en\#!/indicators/008/poten tially-inappropriate-use-of-antipsychotics-in-long-term-care/; mapC1;mapLevel2;/. Accessed 30 Nov 2020.

13. McGreevy J. Dementia and the person-centred care approach. Nursing older people. 2015;27(8).

14. Alzheimer Society. Providing person-centred care. 2020. https:// alzheimer.ca/en/help-support/im-healthcare-provider/providingperson-centred-care. Accessed 30 Nov 2020.

15. BC Patient Safety and Quality Council. Clear Resources. 2020. https://bcpsqc.ca/resources/clear/. Accessed 30 Nov 2020.

16. BC Patient Safety and Quality Council. Final evaluation report Clear wave 1. 2015. https://bcpsqc.ca/wp-content/uploads/2018/ 03/CLeAR-Final-Report-sm.pdf. Accessed 30 Nov 2020.

17. BC Patient Safety and Quality Council. Final evaluation report Clear wave 2. 2017. https://bcpsqc.ca/wp-content/uploads/2018/ 07/2017-CLeAR-Wave-2-Evaluation-Report-Aug-3-2017_ FINAL.pdf. Accessed 30 Nov 2020.

18. Dolan P. The measurement of health-related quality of life for use in resource allocation decisions in health care. Handb Health Econ. 2000;1:1723-60.

19. Canadian Agency for Drugs and Technologies in Health. Guideline for the economic evaluation of health technologies, 4th edition. https://www.cadth.ca/sites/default/files/pdf/cp0008-guide lines-for-economic-evaluation-of-health-technologies.pdf. Accessed 30 Nov 2020.

20. Weinstein MC, Siegel JE, Gold MR, Kamlet MS, Russell LB. Recommendations of the panel on cost-effectiveness in health and medicine. JAMA. 1996;276(15):1253-8.

21. Canadian Institute for Health Information. Potentially Inappropriate Use of Antipsychotics in Long-term Care. 2020. https:// indicatorlibrary.cihi.ca/display/HSPIL/Potentially+Inappropri ate+Use+of+Antipsychotics+in+Long-Term+Care. Accessed 30 Nov 2020.

22. Carpenter I, Hirdes JP. Using interRAI assessment systems to measure and maintain quality of long-term care. A Good Life Old Age. 2013;17:93-139.

23. Canadian Institute for Health Information. Discharge abstract database (DAD). 2020. https://www.cihi.ca/en/discharge-abstr act-database-metadata-dad. Accessed 30 Nov 2020.

24. Canadian Institute for Health Information. National Ambulatory Care Reporting System metadata (NACRS). 2020. https://www. cihi.ca/en/national-ambulatory-care-reporting-system-metadatanacrs. Accessed 30 Nov 2020.

25. Population data BC. PharmaNet Dataset. 2020. https://www.popda ta.bc.ca/data/health/PharmaNet. Accessed 30 Nov 2020.

26. Population data BC. Medical Services Plan Dataset. 2020. https:// www.popdata.bc.ca/data/health/msp. Accessed 30 Nov 2020.

27. Institute of Health Economics. Estimating costs of hospital stays. 2015. https://www.ihe.ca/publications/estimating-costs-of-hospi tal-stays. Accessed 30 Nov 2020.

28. Ministry of Health. Medical Services Commission Payment Schedule. 2020. https://www2.gov.bc.ca/assets/gov/health/pract itioner-pro/medical-services-plan/msc-payment-schedule-may2020.pdf. Accessed 30 Nov 2020.

29. Wooldridge JM. Cluster-sample methods in applied econometrics. Am Econ Rev. 2003;93(2):133-8.

30. Dieleman JL, Templin T. Random-effects, fixed-effects and the within-between specification for clustered data in observational health studies: a simulation study. PLoS ONE. 2014;9(10):e110257.

31. Health Employers Association of BC. Collective Agreement 2019-2022. 2020. http://www.heabc.bc.ca/public/CAs/APAD/ 2019-2022APADBA_CA.pdf. Accessed 30 Nov 2020.

32. Hirdes JP, Bernier J, Garner R, Finès P, Jantzi M. Measuring health related quality of life (HRQoL) in community and facility-based care settings with the interRAI assessment 
instruments: development of a crosswalk to HUI3. Qual Life Res. 2018;27(5):1295-309.

33. Moriarty F, Cahir C, Bennett K, Fahey T. Economic impact of potentially inappropriate prescribing and related adverse events in older people: a cost-utility analysis using Markov models. BMJ Open. 2019;9:1.

34. Statistics Canada. Mortality rates by age group. 2020. https:// www150.statcan.gc.ca/t1/tbl1/en/tv.action?pid=1310071001\& pickMembers $\% 5 \mathrm{~B} 0 \% 5 \mathrm{D}=1.11 \&$ pickMembers $\% 5 \mathrm{~B} 1 \% 5 \mathrm{D}=3.1 \&$ cubeTimeFrame. start Year $=2015 \&$ cubeTimeFrame.endYear $=$ 2019\&referencePeriods $=20150101 \% 2$ C20190101. Accessed 30 Nov 2020.

35. TreeAge. 2020. https://www.treeage.com. Accessed 30 Nov 2020.

36. Statistics Canada. Life expectancy in Canada. 2020. https:// www150.statcan.gc.ca/t1/tbl1/en/tv.action?pid=1310040901. Accessed 5 Feb 2021.

37. Mervin MC, Moyle W, Jones C, Murfield J, Draper B, Beattie E, Shum DH, O'Dwyer S, Thalib L. The cost-effectiveness of using PARO, a therapeutic robotic seal, to reduce agitation and medication use in dementia: findings from a cluster-randomized controlled trial. J Am Med Dir Assoc. 2018;19(7):619-22.

38. Zwijsen SA, Bosmans JE, Gerritsen DL, Pot AM, Hertogh CM, Smalbrugge M. The cost-effectiveness of grip on challenging behaviour: an economic evaluation of a care programme for managing challenging behaviour. Int J Geriatr Psychiatry. 2016;31(6):567-74.
39. Jódar-Sánchez F, Martín JJ, López-del-Amo MP, García L, Araújo-Santos JM, Epstein D. Cost-utility analysis of a pharmacotherapy follow-up for elderly nursing home residents in Spain. J Am Geriatrics Soc. 2014;62(7):1272-80.

40. Luxford K, Safran DG, Delbanco T. Promoting patient-centered care: a qualitative study of facilitators and barriers in healthcare organizations with a reputation for improving the patient experience. Int J Qual Health Care. 2011;23(5):510-5.

41. Berta W, Laporte A, Kachan N. Unpacking the relationship between operational efficiency and quality of care in Ontario long-term care homes. Can J Aging/La Revue Canadienne du Vieillissement. 2010;29(4):543-56.

42. Rosenthal MB, Fernandopulle R, Song HR, Landon B. Paying for quality: providers' incentives for quality improvement. Health Aff. 2004;23(2):127-41.

43. Alberta Health Services. Appropriate use of antipsychotics in dementia. 2017. https://www.albertahealthservices.ca/assets/ about/scn/ahs-scn-srs-aua-fuss-notes.pdf. Accessed 30 Nov 2020.

44. Canadian Foundation for Healthcare Improvement. Appropriate use of antipsychotics. 2109. https://www.cfhi-fcass.ca/what-wedo/spread-and-scale-proven-innovations/appropriate-use-of-antip sychotics. Accessed 30 Nov 2020.

\title{
Authors and Affiliations
}

\author{
Asif Raza Khowaja ${ }^{1} \cdot$ Christina Krause $^{2} \cdot$ Colleen Kennedy $^{3} \cdot$ Ben Ridout $^{4} \cdot$ Sarah Carriere $^{5} \cdot$ Craig Mitton $^{6}$ \\ Christina Krause \\ ckrause@bcpsqc.ca \\ Colleen Kennedy \\ ckennedy@bcpsqc.ca \\ Ben Ridout \\ BRidout@bcpsqc.ca \\ Sarah Carriere \\ scarriere@bcpsqc.ca \\ Craig Mitton \\ craig.mitton@ubc.ca \\ 1 Department of Health Sciences at Brock University, 1812 Sir \\ 2 BC Patient Safety \& Quality Council and School \\ of Population \& Public Health, Faculty of Medicine \\ at the University of British Columbia, Vancouver, Canada \\ 3 Health System improvement, BC Patient Safety \& Quality \\ Council, Vancouver, Canada \\ 4 Analytics and Strategic Initiatives, BC Patient Safety \& \\ Quality Council, Vancouver, Canada \\ 5 Health Systems Improvement, BC Patient Safety \& Quality \\ Council, Vancouver, Canada \\ 6 School of Population and Public Health; and Senior Scientist \\ at the Centre for Clinical Epidemiology and Evaluation, \\ Vancouver, Canada
} Isaac Brock Way, St. Catharines, ON L2S 3A1, Canada 